Original Research Paper

\title{
Separation and Identification of Two Fungal Strains in Stored Maize
}

\author{
${ }^{1,2}$ Xiaoyu Yue, ${ }^{2}$ Lynne McLandsborough, ${ }^{1}$ Jian Zou and ${ }^{1}$ Xueqin Gao \\ ${ }^{I}$ College of Food Engineering, Henan University of Animal Husbandry and Economy, Zhengzhou Henan 450046, China \\ ${ }^{2}$ Department of Food Science, University of Massachusetts, Amherst, MA 01003, USA
}

\author{
Article history \\ Received: 06-07-2017 \\ Revised: 22-07-2017 \\ Accepted: 01-08-2017 \\ Corresponding author: \\ Xiaoyu Yue \\ College of Food Engineering, \\ Henan University of Animal \\ Husbandry and Economy, No.6 \\ Longzihu North Road, \\ Zhengdong New District, \\ Zhengzhou, Henan 450046, \\ China \\ E-mail: yuerain@163.com
}

\begin{abstract}
The microbiological spoilage of stored grain is important for human health and economic loss. Two major predominant grain spoilage fungi (M15 and M16) were isolated from maize from a granary in China. Morphological observation of the fungal isolates, analysis of their ITS sequences and construction of their phylogenetic trees were conducted on these two strains, which were identified as Fusarium solani (M15) and Aspergillus sclerotiorum (M16). This method was shown to be a feasible, accurate and convenient way to identify fungi in stored grain.
\end{abstract}

Keywords: Maize, Morphological Characteristics, Analysis of ITS Sequence, Phylogenetic Tree

\section{Introduction}

Stored maize may be contaminated by a variety of microorganisms. Some microbes can produce toxins under appropriate conditions. These toxins may not only lead to worse storage stability and safety but also to a severe threat to human health and lives. Among the microorganisms in stored maize, fungi are the most important, serious and harmful contaminants (Yue et al., 2012; Huang et al., 2010). Thus, the level and variety of fungi can be indicative of the safety of stored maize.

Isolating and identifying the fungi in stored maize that may spoil food and cause disease are important steps in promoting safe storage and preventing fungal toxin hazards to humans. The rDNA Internal Transcribed Spacer (ITS) sequence is highly conserved within strains of the same genus but greatly variable within strains of different genera. This provides abundant genetic information for research in mycology and fungal identification (Agarwal et al., 2008). Along with the development of molecular biology techniques, ITS sequence analysis has been widely used in taxonomy and identification of fungal species because of its good stability and sequencing convenience (Li et al., 2008; Geml et al., 2009; Alaei et al., 2009; Tedersoo et al. 2009).

Previously, we have reported the characterization of two of the spoilage fungi (Yue et al., 2011; 2014) and in this study, two predominant fungi that spoil food (M15 and M16) were isolated and purified from the stored maize collected from a granary in China. Morphological observation and analysis of ITS sequence were conducted on these two strains and phylogenetic trees were constructed to identify M15 and M16. The results showed that the two strains were Fusarium solani (M15) and Aspergillus sclerotiorum (M16). The taxonomic identification method used in our study was more reliable and convenient than the traditional method of fungal classification, which is mainly based on morphological characteristics, growth characteristics and comparison of physiological and biochemical indicators with those of standard strains. Also, this study provides references for future research in fungal diversity and the safety of stored grain.

\section{Materials and Methods}

\section{Materials}

The maize used in this study was collected from a granary in Henan Province, China. These samples were saved at $4^{\circ} \mathrm{C}$ for later use. Taq DNA polymerase, proteinase $\mathrm{K}$, RNase enzyme were bought from TIANGEN Biotech, Beijing, China. All the primers used for PCR testing were bought from Sangon Biotech, Shanghai, China. 
Isolation, Purification and Preservation of the Fungal Strains

Three maize samples of approximately $25 \mathrm{~g}$ were placed into sterilized Petri dishes. Sterile water was added to maize to bring the water activity to 0.97 and these samples were incubated at $28^{\circ} \mathrm{C}$ and water activity 0.97. After mold colonies became visible, they were isolated and purified on potato dextrose agar (PDA, Land Bridge Technology, Beijing, China) at $28^{\circ} \mathrm{C}$. Stocks of purified cultures were grown on PDA and saved at $4^{\circ} \mathrm{C}$ for later use.

\section{Morphological Observation of the Fungal Strains}

Morphological observation of the selected isolates were characterized on PDA medium, Czapek agar (CA, Land Bridge Technology, Beijing, China) and Czapek yeast agar (CYA, Land Bridge Technology, Beijing, China). These plates were cultivated at $28^{\circ} \mathrm{C}$ for 12 days for morphological observation of the fungal isolates. Colors, shapes, sizes of the colonies and density of mycelia in the medium were observed.

The insert cultivation method was used to observe hyphae and spore morphology (Yue et al., 2014). The strains were insert-cultivated for 3-5 days. The cover glass was taken out for microscopic observation after hyphae had spread onto the glass. Spore morphology, the morphological structure of the spore head and the presence or absence of septa within the hyphae were observed using a CX 21 microscope (Olympus Corp.) connected to a $600 \mathrm{D}$ camera (Canon Inc.).

\section{DNA Preparation of the Strains}

The activated strains from preservation of the fungal strains were inoculated into $200 \mathrm{~mL}$ liquid medium in a triangular flask, which was put into a shaker for cultivation at $28^{\circ} \mathrm{C}$ and $180-200 \mathrm{rpm}$ for $96 \mathrm{~h}$. Then the collected hyphae of the strains were observed with a microscope so that their purity was tested.

The culture solution was filtered with sterile gauze (2 layers). Then the gauze was washed with sterile deionized water 2-3 times and with TE buffer (10mmol//Tris-HCI, lmmol/1 EDTA, $\mathrm{pH} 8.0)$ once. The collectedly dry hyphae was stored at $20^{\circ} \mathrm{C}$ for later use.

The CTAB method was used for extracting the total DNA of the sample strains. Previous methods were reviewed (Schabereiter-Gurtner et al., 2001; Wei, 2005). The extracted DNA samples ( $5 \mathrm{uL}$ for each sample) were visualized by electrophoretic separation in an $1 \%$ agarose gel which was stained with Goldview (SBS Genetech Co., Ltd., Beijing, China) and visualized utilizing UV light to evaluate extraction quality prior to amplification.

\section{Amplification of the ITS Regions}

The extracted DNA samples were amplified with PCR reaction for ITS regions. The forward primer (ITS1-5.8S, 5'-3') was GGAAGTAAA
AGTCGTAACAAGG. The reverse primer (rDNA-ITS2, 5'-3') was TCCTCCGCTTATTGATATGC (Wei, 2005). The PCR reaction was heated for $5 \mathrm{~min}$ at $95^{\circ} \mathrm{C}$, followed by 40 cycles at $94^{\circ} \mathrm{C}$ for $30 \mathrm{~s}$, at $55^{\circ} \mathrm{C}$ for $30 \mathrm{~s}$ and at $72^{\circ} \mathrm{C}$ for $40 \mathrm{~s}$ and then heated at $72^{\circ} \mathrm{C}$ for $7 \mathrm{~min}$. The amplification products were stored at $-20^{\circ} \mathrm{C}$.

The PCR products $(5 \mathrm{uL}$ for each sample) were separated in $2 \%$ agarose gels, dyed with ethidium bromide and visualized utilizing UV light and documented using a JS-1070P gel documentation systems (Peiqing Co., Ltd, Shanghai, China) to confirm size and quality of the amplification products. A standard DNA marker (SOURCE) was used to estimate DNA length.

\section{Sequencing of the ITS Regions and Phylogenetic Analysis}

Sequencing of the ITS1 region PCR products was conducted by Sangon Biotech, Shanghai, China, utilizing the primers from PCR. The sequencing results were compared with sequences in the BLAST (Basic Local Alignment Search Tool) GenBank (https://blast.ncbi.nlm.nih.gov/Blast.cgi). Reference sequences with high homology were collected from the BLAST GenBank. Phylogenetic analysis was performed with Molecular Evolutionary Genetic Analysis (MEGA), version 5.05 (Saitou and Nei, 1987; Tamura et al., 2011) with the Neighbor-Joining method and a bootstrap value 1000 .

\section{Identification of the Fungal Strains}

Morphological observation, analysis of ITS sequence and phylogenetic analysis were combined to identify the fungal strains. Two manuals (WC, 1973; Wei, 1979) about fungal identification were also consulted.

\section{Results}

\section{Isolation and Purification of Predominant Fungi}

After inoculating and cultivating, several different types of colonies with different morphologies were observed on PDA medium plates. These strains were identified as fungi that presumptively spoil grain and four colony morphologies were observed. Previously, we have reported the characterization of two of the spoilage fungi Aspergillus oryzae and Chaetomium globosum (Yue et al., 2011; 2014) and among the predominant colonies, M15 and M16 were separated and purified. (Pictures were not shown)

\section{Morphological Characteristics of M15 and M16}

The microscopy results and colony morphology of the strain M15 are shown in Fig. 1-4. Dense hyphae with septa were observed in M15 under microscope. Microconidia were scattered, transparent and smooth and their shape was oval or ovoid Fig. 1. The colony diameter of M 15 was $60 \mathrm{~mm}$ after 7 days of cultivation 
in PDA medium at $27^{\circ} \mathrm{C}$, while the diameter was $80 \mathrm{~mm}$ after 12 days. Aerial hyphae were white, flocculent and flat Fig. 2, (PDA). After 7 days of cultivation in CA medium at $27^{\circ} \mathrm{C}$, the colony diameter of M15 was $60-65$ $\mathrm{mm}$, while the diameter was about $80 \mathrm{~mm}$ after 12 days.

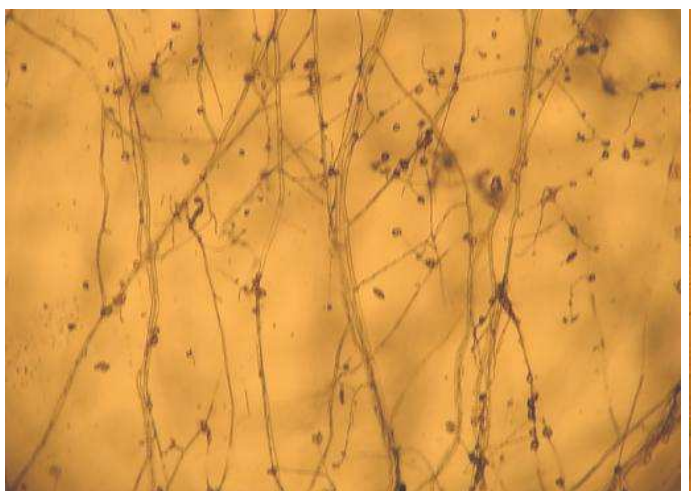

(a)
Aerial hyphae were white and canary yellow and a bluegray concentric circle was formed around the colony Fig. 2 (CA). The colony diameter was $70 \mathrm{~mm}$ after 7 days of cultivation in CYA medium at $27^{\circ} \mathrm{C}$. Aerial hyphae were white and dense Fig. 2 (CYA).

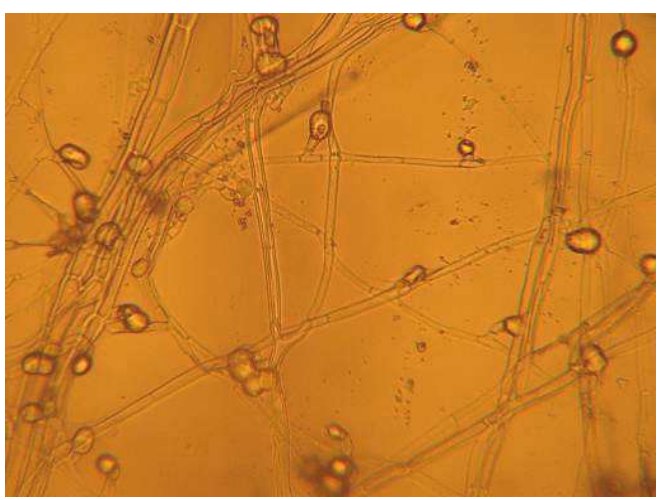

(b)

Fig. 1. The microscopy results of the strain M15 (a) The picture was magnified 100 times (b) The picture was magnified 400 times

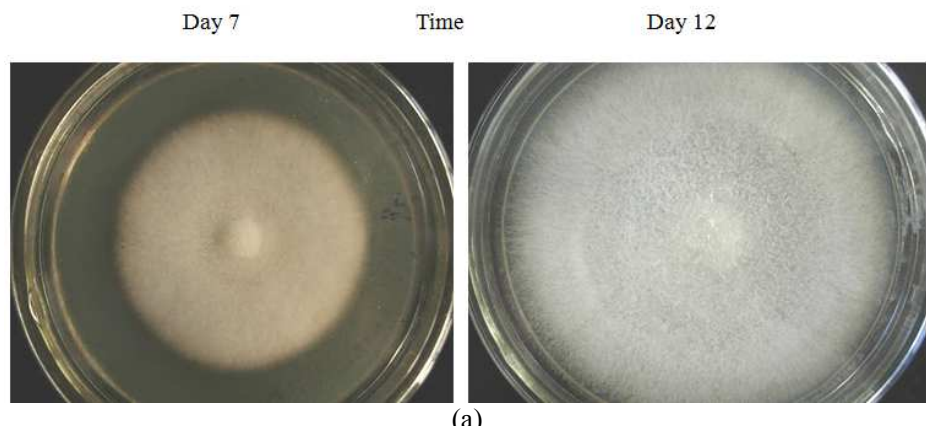

(a)
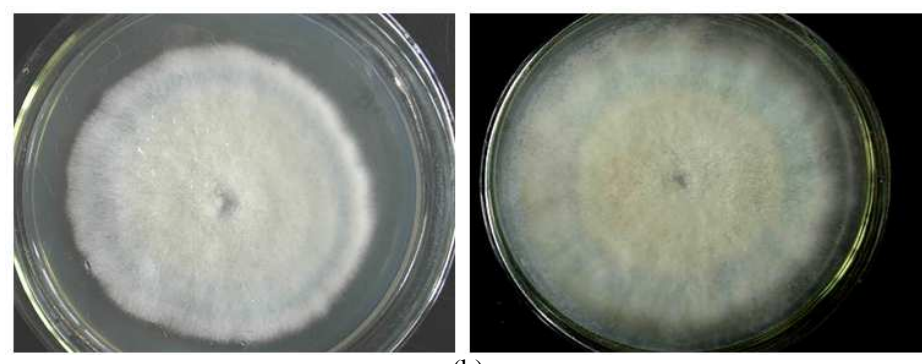

(b)
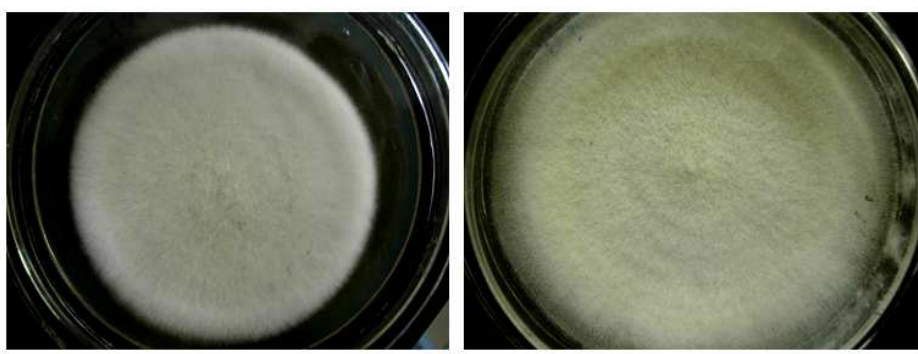

(c)

Fig. 2. Appearance of colonies of the strain M15 on PDA, CA and CYA medium at 7 and 12 days at $27^{\circ} \mathrm{C}$ (A) colonies were grown on PDA (B) on CA and (C) on CYA media 


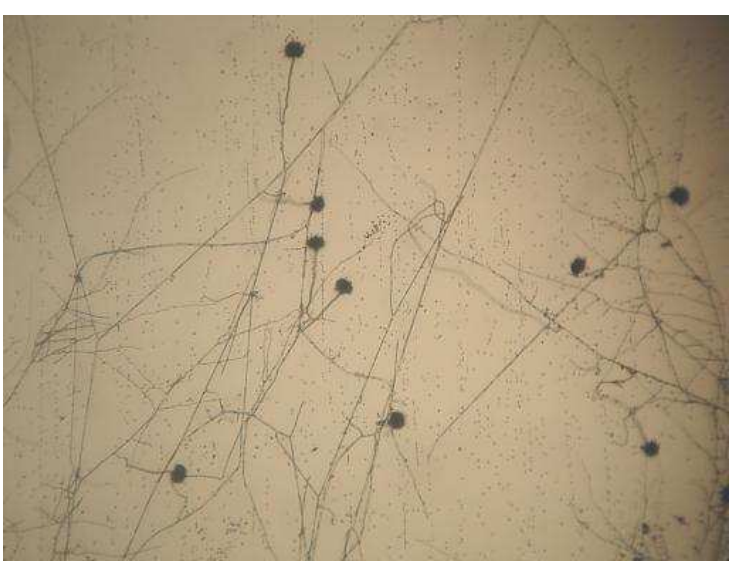

(a)

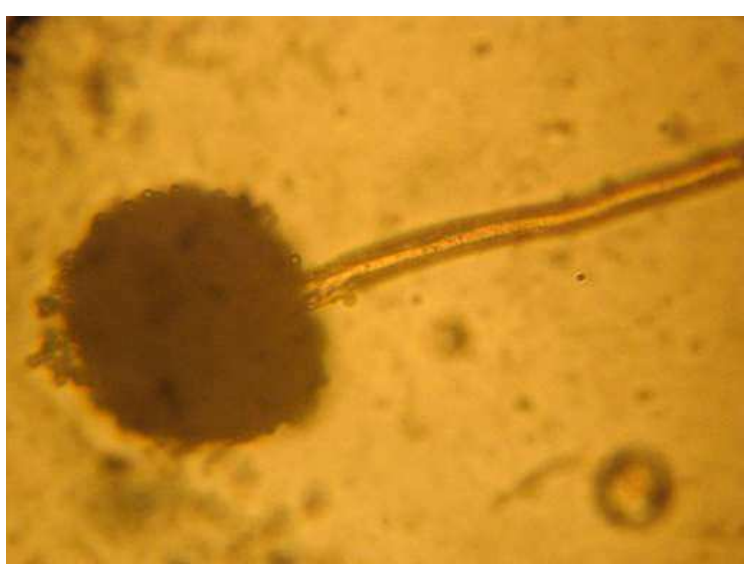

(b)

Fig. 3. The microscopy results of the strain M16 (a) the picture was magnified 100 times (b) the picture was magnified 400 times

Day 7

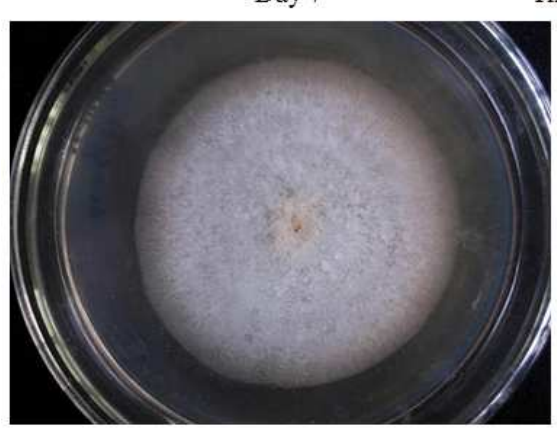

Time

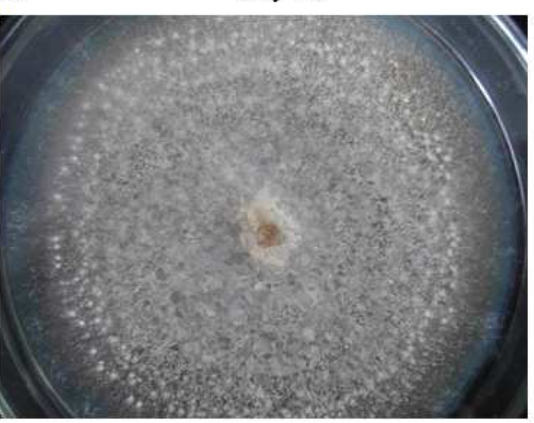

(a)
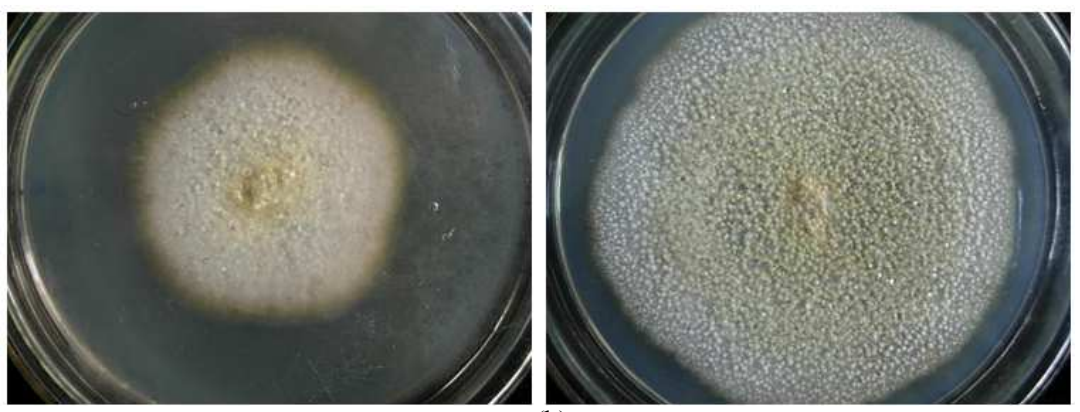

(b)
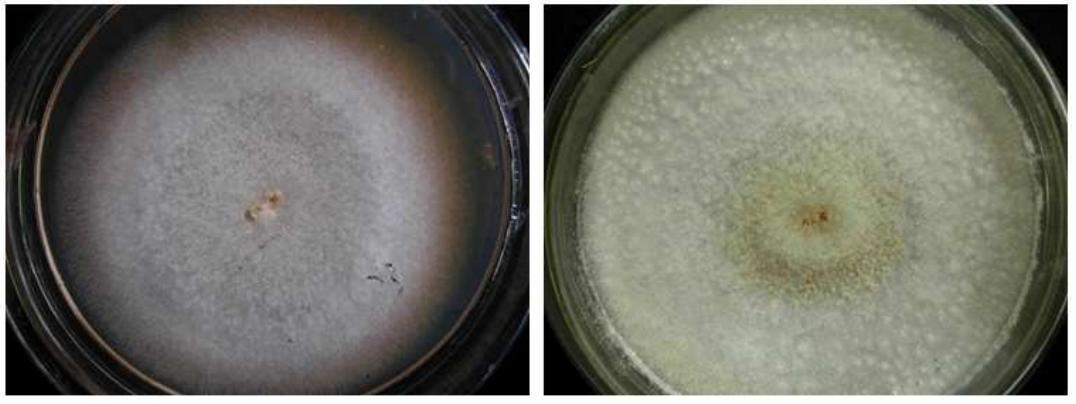

(c)

Fig. 4. Appearance of colonies of the strain M16 on PDA, CA and CYA medium at 7 and 12 days at $27^{\circ} \mathrm{C}$ (A) colonies were grown on PDA, (B) on CA and (C) on CYA media 


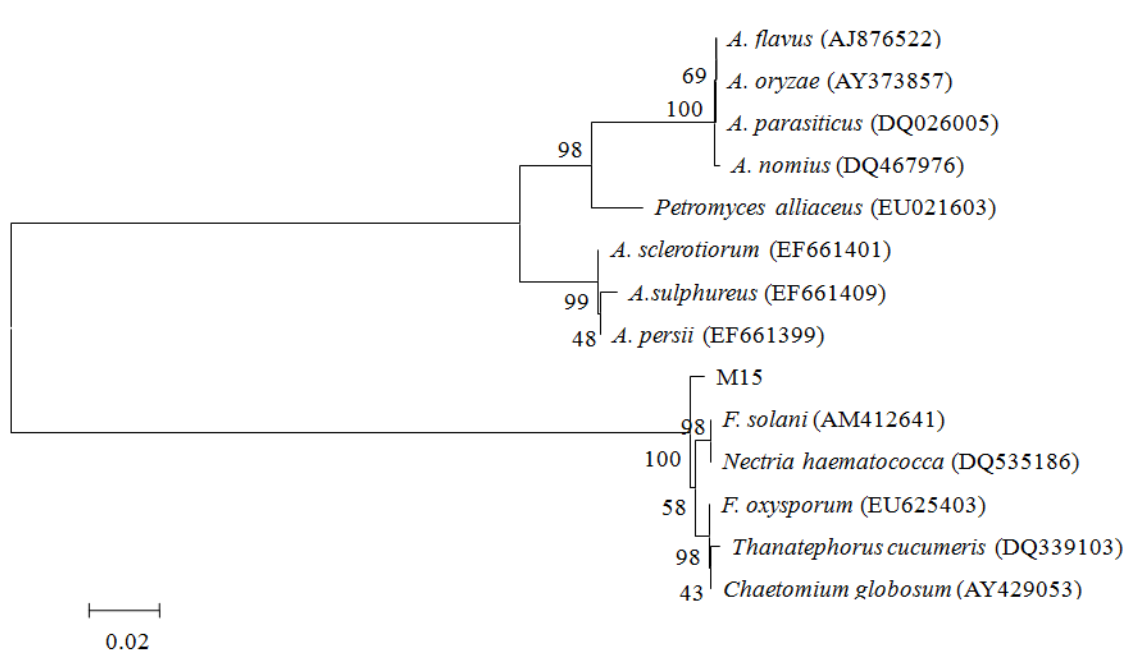

(a)

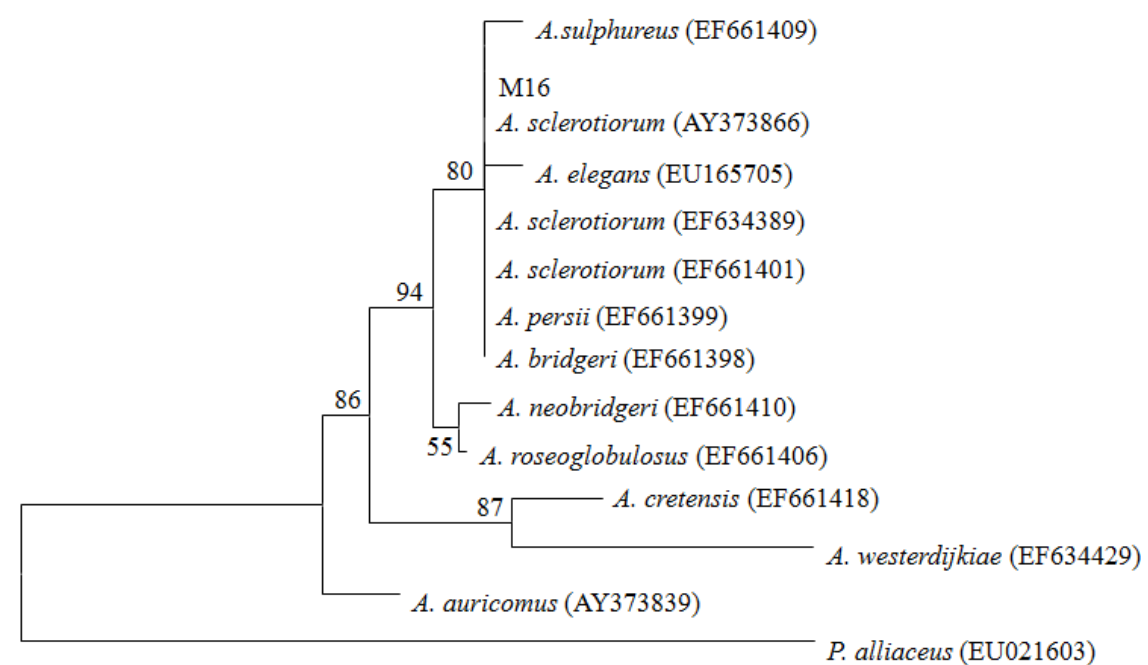

$\mapsto$

0.001

(b)

Fig. 5. The phylogenetic tree based on a comparison of the ITS rDNA sequence between the test strain M15 (A) or M16 (B) and selected reference strains. Bootstrap value was 1000 and Kimura 2-parameter model was used

The microscopy results and colony morphology of the strain M16 are shown in Fig. 3-4. The conidial head of M16 was round Fig. 3a with the development conidiophores Fig. 3b. When grown on PDA medium, the colony diameter of the strain M16 was $45 \mathrm{~mm}$ after 7 days and 65-75 mm after 10-12 day Fig. 4a. The colony color was white. Sclerotia were granular, spherical or spheroidal. The arrangement of the sclerotia was as concentric circles Fig. 4a. The colony diameter of M16 was $37 \mathrm{~mm}$ after 7 day of cultivation on CA medium at $27^{\circ} \mathrm{C}$, while the diameter was about $70-80 \mathrm{~mm}$ after 10 12 day. Hyphae were yellowish green and velvety in texture. Many sclerotia were produced and granular in appearance. The color of sclerotia was white at first, but later it gradually became yellowish green Fig. $4 b$. The colony diameter was $70 \mathrm{~mm}$ after 7 days of cultivation in CYA medium at $27^{\circ} \mathrm{C}$. Texture of the colonies was velvety. The colony color was white at first, but it gradually became pale yellow later. Many sclerotia were formed Fig. 4c.

\section{Homologous Analysis of ITS Sequence of the Strains}

The ITS sequences of each strain was amplified using PCR and the approximate length of the ITS regions for M15 and M16 were approximated using gel electrophoresis to 560 and $590 \mathrm{bp}$, respectively. The PCR samples for the ITS regions of M15 and M16 were 
sequenced. The sequence of the amplified M15 ITS sequence was $565 \mathrm{bp}$ and included full sequences of ITS1 5.8S and ITS2, as well as a partial sequence of $28 \mathrm{~S}$ rDNA. BLAST results of the sequence were compared in DNAMAN (Version 5.2.2) for similarity. The phylogenetic tree for M15 in Fig. 5A. It was observed that Fusarium solani (AM412641), Nectria haematococca (DQ535186), F. oxysporum (EU625403), Thanatephorus cucumeris (DQ339103) and Chaetomium globosum (AY429053) had relatively less evolutionary distance from M15 than did the other selected reference strains Fig. 5a. F. solani (AM412641) had the highest sequence homology with M15, which was $98.3 \%$. Using morphological observation (WC, 1973; Wei, 1979) along with ITS sequence and phylogenetic analysis were all considered, we conclude that M15 is a F. solani isolate.

The ITS region of isolate M16 was sequenced and the $604 \mathrm{bp}$ included a partial 18S rDNA sequence, full sequences of ITS1 5.8S and ITS2 and a partial sequence of $28 \mathrm{~S}$ rDNA. BLAST results were compared in DNAMAN. The results showed that A. sclerotiorum (AY373866) had the highest sequence homology with M16, which was $100 \%$ Fig. 5 b.

A. bridgeri (EF 661398), A. sclerotiorum (AY 373866) and $A$. persii (EF 661399) had higher phylogenetic homology with M16 than did the other selected reference strains Fig. 5b. Using morphological observation (WC, 1973; Wei, 1979) along with ITS sequence and phylogenetic analysis were all considered, we conclude that M16 is an A. sclerotiorum isolate.

\section{Discussion}

In previous papers on identifying fungal strains in maize under different storage conditions, predominant genera were Penicillium, Aspergillus, Rhizopus and Fusarium. Specific fungal species in maize were also identified previously (Xu et al., 2015; Cheng and Chen, 2011; Yue et al., 2014; 2011). Many fungi can influence the quality and the safety of stored maize.

The genus Fusarium (Nectriaceae, Hypocreales, Ascomycetes) includes many species, which are widespread and metatrophic. Some of them can cause serious diseases of wheat, maize, rice, vegetables, etc. Some of them are even pathogens for people and animals. Fusarium solani is one of the most frequently isolated fungi from grain, soil and plant debris. It is also ubiquitous in soil and decaying plant material, where it is important in decompostion. It is a host-specific pathogen of a number of agriculturally important plants (Booth, 1971; Summerbell, 2003; Zhang et al., 2006). Fusarium solani mainly infects plants by wound infection and it has a strong parasitic ability and pathogenicity through soil (Wang et al., 2000). It can harm many kinds of crops, cause diseases, reduce yield and lower the quality of crops. Fusarium solani is also one of the main pathogenic microorganisms of lawn diseases (Zhang, 2010). At the same time, it can also cause fungal corneal disease in humans (Wang et al., 2003).
Aspergillus is widespread in nature. It can be found in soil, putrid organic matter, stored grain and all kinds of food. Aspergillus sclerotiorum is one of the most common molds on food and grain and it is the main cause of grain and food spoilage. Five secondary metabolites (sclerotiamide, scleramide, penicillic acid, ochratoxin A and Ro 09-1469) have been previously reported from A. sclerotiorum (Zheng et al., 2009) and A. sclerotiorum is known to produce ochratoxin A when grown on pearled wheat (Whyte et al., 1996).

Fungi in stored maize can cause grain spoilage and their toxins hazard to humans. Therefore, a rapid and reliable identification method is important in this area. With the advancement of technology, DNA sequence analysis for microorganism identification has become feasible and convenient. In this study, the predominant fungi in stored maize were identified combining morphological observation of the fungal isolates, analysis of their ITS sequences and construction of their phylogenetic trees. Two predominant fungi (M 15 and M 16) were identified as Fusarium solani and Aspergillus sclerotiorum. The feasibility of using morphological and physiological characteristics as well as ITS sequencing for identification of stored fungi was demonstrated. This approach provides a rapid alternative to traditional methods for fungal species delineation. In addition, the number of ITS sequences available in the GenBank database has increased rapidly in recent years. The expanding database has improved the quality and accuracy of fungal identification (Hinrikson et al., 2005). The current study combined with previous research has proved that this method is effective and feasible for identifying fungal strains.

\section{Acknowledgement}

The authors thank Dr. Wentao $\mathrm{Xu}$ for his advice relating to the molecular biology research.

\section{Funding Information}

This work was supported by the National Natural Science Foundation of China (U1 404332); the Science and Technology Innovation Talent Project in Henan Province Colleges and Universities, China (16 HASTIT017); the Project of Science and Technology Innovation Team of the Henan University of Animal Husbandry and Economy (HUAHE 2015015).

\section{Authors' Contributions}

Xiaoyu Yue and Jian Zou: Has contributed with planning and implementation of the research work as well as interpretation of data and article preparation.

Lynne McLandsborough: Has contributed with interpretation of data. 
Xueqin Gao: Has contributed with planning and implementation of research work as well as revising the article critically.

\section{Conflict of Interest}

The authors declare that they have no competing interests. The corresponding author affirms that all of the other authors have read and approved of the manuscript.

\section{References}

Agarwal, M., N. Shrivastava and H. Padh, 2008. Advances in molecular marker techniques and their applications in plant sciences. Plant Cell Reports, 27: 617-631. DOI: 10.1007/s00299-008-0507-z

Alaei, H., M. de Backera, J. Nuytinckc, M. Maesa and M. Höfteb et al., 2009. Phylogenetic relationships of Puccinia horiana and other rust pathogens of Chrysanthemum $\times$ morifolium based on rDNA ITS sequence analysis. Mycological Res., 113: 668-683. DOI: 10.1016/j.mycres.2009.02.003

Booth, C., 1972. The genus fusarium. Mycologia, 64: 1199-1202. DOI: $10.2307 / 3758092$

Cheng, F. and W. Chen, 2011. Study on fungal diversity of maize under different storage conditions. J. Chinese Cereals Oils Assoc., 26: 83-87.

Hinrikson, H.P., S.F. Hurst, T.J. Lott, D.W. Warnock and C.J. Morrison, 2005. Assessment of ribosomal largesubunit D1-D2, internal transcribed spacer 1 and internal transcribed spacer 2 regions as targets for molecular identification of medically important Aspergillus species. J. Clin. Microbiol., 43: 2092-2103. DOI: 10.1128/JCM.43.5.2092-2103.2005

Geml, J., G.A. Laursen, I. Timling, J.M. McFarland and M.G. Booth et al., 2009. Molecular phylogenetic biodiversity assessment of arctic and boreal ectomycorrhizal Lactarius Pers. (Russulales; Basidiomycota) in Alaska, based on soil and sporocarp DNA. Molecular Ecol., 18: 2213-2227. DOI: $10.1111 / \mathrm{j} .1365-294 X .2009 .04192 . \mathrm{X}$

Huang, S.X., J.P. Cai and H.J. Tian, 2010. Mould development characters of different stored grains. J. Chinese Cereals Oils Assoc., 25: 99-102.

Li, Z.H., X.M. Feng, S.Q. Lu, F. Zhang and F.Y. Wang et al., 2008. Molecular phylogeny of Pneumocystis based on 5.8S rRNA gene and the internal transcribed spacers of rRNA gene sequences. Sci. China Series C Life Sci., 51: 445-452. DOI: $10.1007 / \mathrm{s} 11427-008-0057-1$

Schabereiter-Gurtner, C., G. Pinar, W. Lubitz and S. Rolleke, 2001. An advanced molecular strategy to identify bacterial communities on art objects. J. Microbiol. Meth., 45: 77-87.

Saitou, N. and M. Nei, 1987. The neighbor-joining method: A new method for reconstructing phylogenetic trees. Molecular Biol. Evolution, 4: 406-425.
Summerbell, R.C., 2003. Aspergillus, Fusarium, Sporothrix, Piedraia and Their Relatives. In: Pathogenic Fungi in Humans and Animals, Howard, D.H. (Ed.), CRC Press, New York, ISBN-10: 0203909100, pp: 237-498.

Tamura, K., D. Peterson, N. Peterson and G. Stecher, 2011. Masatoshi nei and sudhir kumar MEGA5: Molecular evolutionary genetics analysis using maximum likelihood, evolutionary distance and maximum parsimony methods. Molecular Biol. Evolution, 28: 2731-2739. DOI: $10.1093 / \mathrm{molbev} / \mathrm{msr} 121$

Tedersoo, L., T. Suvi, T. Jairus, I. Ostonen and S. Põlme, 2009. Revisiting ectomycorrhizal fungi of the genus Alnus: Differential host specificity, diversity and determinants of the fungal community. New Phytologist, 182: 727-735. DOI: $10.1111 /$ j.1469-8137.2009.02792.x

Wang, L.Y., S.T. Sun, Y.Q. Zhang, Y.Q. Wang and L. Zhu et al., 2003. The pathogenic spectrum investigation of fungal keratitis in 1996-2002 of Henan. Chinese J. Practical Ophthalmology, 21: 224-225.

Wang, Y., Y.J. Yang, X.R. Yang, D.L. Yan and R.S. Zhao et al., 2000. Isolation and identification of fusarium solani and fusarium solani var, Coeruleumpathogens of eggplant root rot. Tianjin Agric. Sci., 6: 4-6. DOI: 10.3969/j.issn.1006-6500.2000.03.002

Wei, J.C., 1979. Identification Handbook of Fungi. 1st Edn., Shanghai Science and Technology Press, Shanghai.

Wei, X.X., 2005. Isolation and Identification of Stachybotrys sp. and Property Analysis on Its $\beta$ glucosidase. Unpublished dissertation in partial fulfillment of the requirements. $\mathrm{PhD}$ Thesis Agricultural University, Beijing, China.

Whyte, A.C., J.B. Gloer, D.T. Wicklow and P.F. Dowd, 1996. Sclerotiamide: A new member of the paraherquamide class with potent antiinsectan activity from the sclerotia of aspergillus sclerotiorum. J. Natural Products, 59: 1093-1095. DOI: $10.1021 / \mathrm{np} 960607 \mathrm{~m}$

WC, 1973. Institute of Microbiology, Chinese Academy of Sciences. 1st Edn., Common Fungi Science Press, Beijing.

Xu, Y.Y., J. Yu, B.B. Miao and H.H. Guan, 2015. Separation purification and identification of molds in corn. Food Res. Dev.

Yue, X.Y., Z.X. Hu, J. Xu, Z.G. Li and X. Li et al., 2014. Isolation and identification of a predominant spoiling strain M14 of mold in the stored maize. J. Chinese Inst. Food Sci. Technol., 14: 269-276.

Yue, X.Y., H.Y. Zhang, T. Xin, X. Li and X.D. Liu et al., 2012. Research progress in predictive microbiology models of stored grain. J. Chinese Cereals Oils Assoc., 27: 118-123. 
Yue, X.Y., J. Xue, H.Y. Zhang, Z.W. Sun and X. Li et al., 2011. Isolated and identified of predominant spoiling strain $\mathrm{m} 13$ of mold in the stored maize. Sci. Technol. Food Industry, 32: 183-186.

Zhang, C.X., 2010. Isolation and identification of fusarium spp. from rhizosphere of three turf grasses in kunmning. Grassland Turf, 30: 31-33. DOI: $10.3969 /$ j.issn. 1009-5500.2010.04.007
Zhang, N., K. O’Donnell, D.A. Sutton, F.A. Nalim and R.C. Summerbell et al., 2006. Members of the fusarium solani species complex that cause infections in both humans and plants are common in the environment. J. Clin. Microbiol., 44: 2186-2190.

Zheng, J.K., H.J. Zhu, K. Hong, Y. Wang and P.P. Liu et al., 2009. Novel cyclic hexapeptides from marinederived fungus, aspergillus sclerotiorum PT06-1. Organic Lett., 11: 5262-5265. 